\title{
FRACIONAMENTO FÍSICO DA MATÉRIA ORGÂNICA DE UM LATOSSOLO VERMELHO DISTRÓFICO TÍPICO PELO MÉTODO DE SONICAÇÃO
}

\author{
Physical fractionation of organic matter of a typical distrophic red latosol by the sound method
}

\author{
Rolff Vladimir Mitton ${ }^{1 *}$; Jeidi Yasmin Galeano Cobos²; Leovanio Rodrigues Barbosa ${ }^{1}$; Jana Daisy Honorato Borgo ${ }^{1}$ \\ ${ }^{1}$ Engenheiros Agrônomos, Discentes do Programa de Pós-Graduação em Ciência do Solos da Universidade Federal do \\ Paraná (UFPR), Setor de Ciências Agrárias (SCA), Departamento de Solos e Engenharia Agrícola (DSEA). E-mail * \\ rvm5663@gmail.com. \\ ${ }^{2}$ Engenheira em Agroecologia; Discente do Programa de Pós-Graduação em Agronomia - Produção Vegetal, Departamento de \\ Fitotecnia e Fitossanitarismo, Setor de Ciências Agrárias, Universidade Federal do Paraná.
}

Artigo enviado em 20/11/2016, aceito em 13/05/2017 e publicado em 07/07/2017.

\begin{abstract}
RESUMO - A matéria orgânica do solo (MOS) pode ser definida como todo material que contém carbono orgânico do solo (COS), incluindo os microrganismos, vivos e mortos, resíduos de plantas e animais parcialmente decompostos, produtos da sua decomposição e substâncias orgânicas microbiológicas e/ou quimicamente alteradas. O fracionamento físico da MOS é caracterizado pela sua avaliação de forma menos destrutível comparado com o fracionamento químico. É realizada através de alterações na proporção das frações lábeis, como o carbono da fração grosseira, carbono orgânico particulado (COP), bem como, as frações que estejam associadas aos minerais (COAM), as quais podem fornecer informações importantes sobre a sustentabilidade ambiental e sobre a qualidade do solo. Os resultados obtidos deste fracionamento, que consiste na separação das frações da MOS agrupadas em diferentes tamanhos de partícula, são mais diretamente relacionados com frações que realmente existem no solo. Uma das ferramentas para que a técnica de fracionamento físico da matéria orgânica seja efetuada é a sonicação. O sonicador opera através da vibração ultrassônica, onde ocorre a formação de bolhas microscópicas, pela cavitação, o suficiente para produzir pressão e energia para o rompimento de ligações responsáveis pela agregação das partículas. Neste processo ocorre uma máxima dispersão na superfície das partículas do solo, fendas e linhas de fraqueza, com uma mínima alteração da amostra. A energia liberada para essa dispersão promove o aumento da temperatura da solução com o aumento do

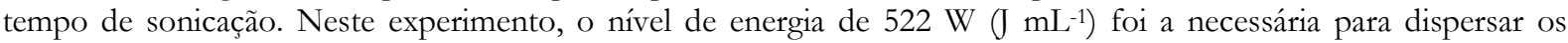
compartimentos da matéria orgânica do solo com maior proporção de carbono $(\mathrm{C})$ associada aos minerais (COAM), e também com menor proporção, associada à fração particulada (COP).
\end{abstract}

Palavras-chave - Carbono orgânico do solo, frações lábeis, energia ultrassônica, estabilidade de agregados.

\begin{abstract}
Soil organic matter (SOM) can be defined as any soil organic carbon (SOC) material, including living and dead micro-organisms, partially decomposed plant and animal waste, decomposition products and organic microbiological substances and / or chemically altered. The physical fractionation of SOM is characterized by its less destructible evaluation compared to the chemical fractionation. It is performed through changes in the proportion of labile fractions, such as coarse fraction carbon, particulate organic carbon (POC), as well as fractions that are associated with minerals (COAM), which can provide important information on environmental sustainability and soil quality. The results obtained from this fractionation consisting of the separation of the fractions of the MOS grouped in different particle sizes are more directly related to the fractions that exist in the soil. One of the techniques of physical fractionation of organic matter is sonication. This technique operates through ultrasonic vibration, where the formation of microscopic bubbles occurs, through cavitation, produces pressure and energy for the rupture of connections responsible for the aggregation of particles. The maximum dispersion on the surface of soil particles, cracks and lines of weakness, with minimal sample change. The required energy released for this dispersion promotes disaggregation by heating the solution. In this work, the energy level of $522 \mathrm{~W}\left(\mathrm{~J} \mathrm{~mL}^{-1}\right)$ was necessary for the dispersion of the various compartments of soil organic matter with a higher proportion of carbon $(\mathrm{C})$ associated with minerals (COAM) and the lesser proportion associated with the particulate fraction (POC).
\end{abstract}

Keywords - Organic soil carbon, labile fractions, ultrasonic energy, aggregate stability. 


\section{INTRODUÇÃO}

No sentido mais amplo, a matéria orgânica do solo (MOS) pode ser definida como todo material que contém carbono orgânico do solo (COS), incluindo os microrganismos vivos e mortos, resíduos de plantas e animais parcialmente decompostos, produtos da sua decomposição e substâncias orgânicas microbiológicas e/ou quimicamente alteradas (BERG; LASKOWSKI, 2006; (SCHMIDT et al., 2011)) em diversos estágios de decomposição, unidas através de interações hidrofóbicas e pontes de hidrogênio, conformando uma estrutura supramolecular (PICCOLO et al., 2001; BURDON, 2001; MUSCOLO et al., 2013).

As alterações provocadas pelo uso ou manejo do solo podem ser quantificadas através do fracionamento físico da MOS. Essa quantificação pode ser realizada através das alterações na proporção das frações lábeis, como o carbono da fração grosseira, carbono orgânico particulado (COP), bem como, as frações que estejam associadas aos minerais (COAM), os quais podem fornecer informações importantes sobre a sustentabilidade ambiental e sobre a qualidade do solo

O fracionamento físico da MOS é caracterizado por ser uma técnica menos destrutível, comparado com o fracionamento químico. Neste primeiro método, é considerada a natureza física da MOS, expressa em escala que vai de molecular a macroscópica. Essa determinação, basicamente envolve uma etapa de dispersão obtida pela agitação mecânica ou vibração da amostra, e outra etapa de separação obtida com base no tamanho e densidade da amostra, empregando uma técnica de peneiramento e sedimentação (DICK et al., 2009; JAGADAMMA; LAL, 2010).

Os resultados obtidos neste fracionamento, que consiste na separação das frações da MOS agrupadas em diferentes tamanhos de partícula, estão mais diretamente relacionados com frações que realmente existem no solo. Entretanto, a distribuição do C nas diferentes frações granulométricas depende de uma adequada dispersão do solo. Na sonicação, através da vibração ultrassônica, a formação de bolhas microscópicas, pela cavitação, produz pressão e energia para o rompimento de ligações responsáveis pela agregação das partículas. Ou seja, objetiva-se a máxima dispersão na superfície das partículas do solo, fendas e linhas de fraqueza, com a mínima alteração da amostra (LUNARDI, 2002). A energia liberada promove a desagregação pelo trabalho, e calor, pelo aquecimento da solução. A quantidade de energia aplicada em Joules por mililitro $\left(\mathrm{J} \mathrm{mL}^{-1}\right)$ pela sonda, deve ser suficiente para desagregar os microagregados em partículas individuais. Indiretamente, sabe-se que quanto maior a energia aplicada, maior é a quantidade de argila dispersa em água, e esta pode ser maior quanto maior a estabilidade de seus microagregados.

Entretanto, a sonicação apresenta limitações, como a possibilidade de ruptura de partículas simples de minerais, bem como de micas e biotitas, e possibilidade de ruptura de partículas de MOP e redistribuição para as classes de menor tamanho (LÜTZOW et al., 2007).

Outras técnicas de separação densimétrica mais detalhadas são utilizadas no fracionamento físico, e visam a separação da fração livre e leve (FLL), da matéria orgânica particulada (MOP) externa aos agregados, e da fração leve oclusa (FLO), da MOP oclusa entre os agregados, podendo indicar o efeito da dinâmica da adição de $\mathrm{C}$ pelo manejo do solo sobre estas frações mais grosseiras, sendo ainda utilizadas como técnica para diminuir os erros e aumentar a recuperação do $C$ nas frações após sonicação (SÁ et al., 2005). A FLL e FLO não serão separadas neste trabalho, sendo dada maior atenção à correta calibração do ultrassom e identificação dos níveis de energia sobre a amostra.

A demanda de energia necessária para dispersão dos agregados varia em função do horizonte do solo utilizado, da classe de solo (HANKE, 2012), do teor de MOS, do efeito do manejo aplicado sobre o solo e dos teores de óxidos de Ferro (Fe) e Alumínio (Al) presentes no solo (INDA JUNIOR et al., 2007).

O êxito no tratamento do solo com ultrassom depende não somente das características do solo, mas também das especificações do instrumento e do processo experimental. Entre os fatores que afetam a eficiência da dispersão, encontra-se o decréscimo da eficiência com o aumento da temperatura do líquido, cujo efeito é a redução da cavitação (RAINE; SO, 1994; (KAISER et al., 2012). Desta forma, é de suma importância a calibração da energia efetivamente aplicada pela sonda através da aferição da temperatura da água, pois esta é a energia efetiva produzida por cada aparelho. Além disso, a produção de energia varia não somente entre fabricantes e modelos de aparelho, mas também, em instrumentos de mesma marca e modelo (LUNARDI, 2002).

Este trabalho objetiva descrever a calibração do ultrassom para a dispersão dos agregados de um Latossolo Vermelho Distrófico típico com alto teor de matéria orgânica, através da identificação dos níveis de energia ultrassônica mais adequados para detectar a estabilidade de agregados, e da localização da maior proporção de carbono nos diferentes compartimentos da MOS. 


\section{MATERIAL E MÉTODOS}

\section{Procedência da amostra de solo}

A amostra de solo foi coletada no mês de setembro de 2015 na área experimental (Figura 1.), na Fazenda Experimental Canguiri (CEEx), da Universidade Federal do Paraná (UFPR), localizada no município de Pinhais, Paraná, Brasil. Com as coordenadas geográficas: $25^{\circ} 23^{\prime} 12.3^{\prime \prime S}$ de latitude, $49^{\circ} 07^{\prime} 33.2^{\prime \prime} \mathrm{W}$ de longitude e $920 \mathrm{~m}$ de altitude. O clima da região é classificada como $\mathrm{Cfb}$, segundo Köppen, mesotérmico úmido, com precipitação média mensal de $115,3 \mathrm{~mm}$, temperatura mínima de $15^{\circ} \mathrm{C}$, e máxima de $24,7^{\circ} \mathrm{C}$

A área experimental é altamente mecanizada pelo preparo de solo para cultivo de milho no verão e nabo e/ou aveia no inverno, sendo adubada principalmente com esterco bovino, procedente da produção leiteira da fazenda. O terreno apresenta relevo suave ondulado, localizado em fundo de vale.

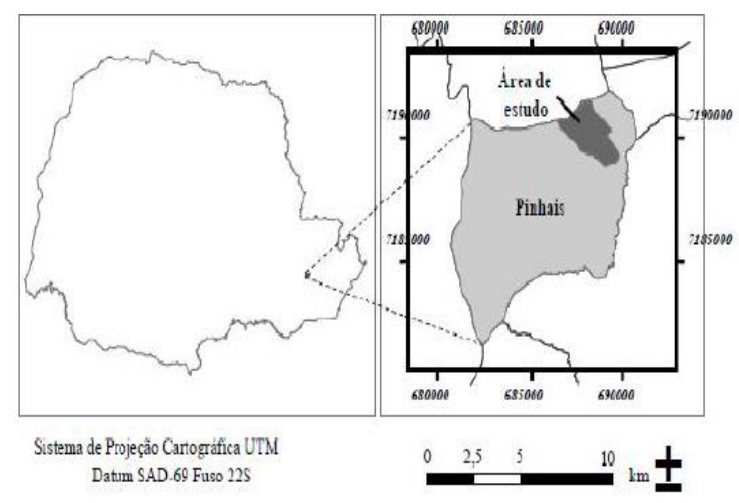

Figura 1 - Localização da procedência do solo, Fazenda Experimental Canguiri (CEEx), Universidade Federal do Paraná (UFPR), município de Pinhais, PR (PRATES et al., 2012).

\section{Fracionamento da matéria orgânica Coleta da amostra de solo}

O solo foi coletado da camada superficial de 0 $20 \mathrm{~cm}$ de profundidade com o auxílio de uma pá. Foi seco ao ar, destorroado e passado em peneira de $2 \mathrm{~mm}$, para obtenção da terra fina seca ao ar (TFSA). O solo foi classificado segundo o Sistema Brasileiro de Classificação de Solos (SiBCS, 3a edição) como um LATOSSOLO VERMELHO Distrófico típico.

\section{Agitação}

Foram agitadas por 16 horas, $20 \mathrm{~g}$ de solo seco em frascos de vidro, com adição de $80 \mathrm{~mL}$ de água comum e sete esferas de acrílico, sendo feitas duas repetições para mitigar qualquer erro aparente durante $o$ experimento. Uma vez finalizado o tempo de agitação, o material foi peneirado a $53 \mu \mathrm{m}$ de diâmetro, separando a fração areia, para obtenção da matéria orgânica particulada (MOP) ligada à fração areia, sendo este o material retido na peneira. E das frações silte e argila, para a obtenção da matéria orgânica humificada (MOH), do $\mathrm{C}$ ligado às frações menores que $53 \mu \mathrm{m}$. A fração areia foi seca em estufa a $50{ }^{\circ} \mathrm{C}$, até apresentar peso constante. Posteriormente, a amostra foi levada ao laboratório de Biogeoquímica do Departamento de Solos e Engenharia Agrícola (DSEA) da Universidade Federal do Paraná (UFPR) para análise de carbono orgânico total (COT) e nitrogênio orgânico total (NOT), pelo método de combustão via seca por meio do aparelho VARIO EL III - Elementar ${ }^{\circledR}$.

A suspensão resultante foi centrifugada a 3000 rpm, em centrífuga HERACUS, durante 10 min. Após a centrifugação, o sobrenadante foi colocado em bécker de $1000 \mathrm{~mL}$, contendo solução de cloreto de cálcio $\left(\mathrm{CaCl}_{2}\right)$, para a floculação total do material a ser sonicado. Dois dias após a floculação, sifonou-se a água, e o material depositado no fundo do bécker foi colocado em um tubo de vidro para ser completado até um volume de $200 \mathrm{~mL}$ (por repetição).

\section{Calibração do Sonicador}

Previamente à sonicação, foi feita a calibração da potência do ultrassom. Para esse etapa, foi sintonizado o aparelho VIBRACEL VCX 700, sendo posteriormente colocado um bécker de vidro, com 200 $\mathrm{mL}$ de água destilada, e aferida a temperatura da água com o uso de um termômetro de coluna de mercúrio, para o registro da temperatura inicial. Após esta etapa, o becker foi colocado dentro de um recipiente plástico contendo água e gelo. Foi inserida a sonda até uma profundidade de $2 \mathrm{~cm}$ dentro da água do bécker, ajustando a amplitude do sonicador a $20 \%$ por 3 minutos (180 s). Após sonicação, a temperatura foi novamente medida e registrada. O mesmo procedimento foi repetido para as amplitudes $30 \%$, $40 \%, 50 \%, 60 \%, 70 \%$ e $80 \%$ (Figura 2). 


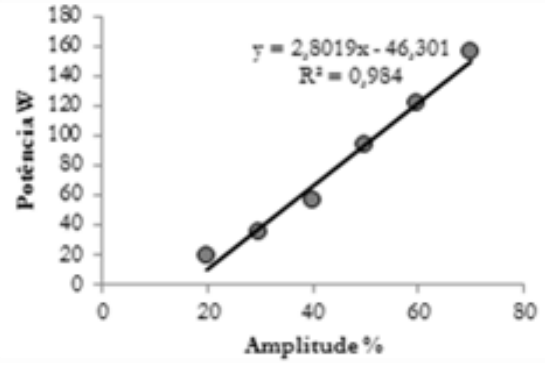

Figura 1 - Curva de calibração da potência.

\section{Potência e energia}

A energia em Joule (J) a ser aplicada na calibração do sonicador foi diretamente proporcional à capacidade calorífica da água, do tempo, e do seu volume. Sendo assim, a energia foi determinada com a seguinte equação:

$\mathbf{E}=\mathbf{C} \cdot \Delta \mathbf{T} \cdot \mathbf{V}$, onde:

$\mathrm{E}=$ Energia $(\mathrm{J})$

$\mathrm{C}=$ Capacidade calorífica $\left(4,183 \mathrm{~J} \mathrm{~mL}^{-1}{ }^{\circ} \mathrm{C}\right)$

$\Delta \mathrm{T}=\mathrm{T}_{\text {final }}-\mathrm{T}_{\text {inicial }}\left({ }^{\circ} \mathrm{C}\right)$

$\mathrm{V}=$ Volume de água $(\mathrm{mL})$

A potência foi uma resultante da relação entre a energia e o tempo, sendo utilizada a equação:

$$
\begin{aligned}
& \mathbf{P}=\mathbf{E} / \mathbf{t} \\
& \mathrm{P}=\text { Potência }(\mathrm{W})=\left(\mathrm{J} \mathrm{s}^{-1}\right) \\
& \mathrm{t}=\text { Tempo }(\mathrm{s})
\end{aligned}
$$

Neste experimento, foi considerada uma amplitude de $50 \%$ para o funcionamento do sonicador, a fim de evitar desgastes excessivos da sonda do ultrassom durante o processo de sonicação. Por esta razão, a equação da curva de calibração foi ajustada da seguinte forma:

$\mathrm{y}=2,8019 \mathrm{x}-46,301$

$\mathrm{y}=2,8019(50)-46,301$

$\mathrm{y}=93,77 \mathrm{~W} \mathrm{ou} \mathrm{J} \mathrm{s}^{-1}$

\section{Curva de dispersão da argila}

Para a realização da curva de dispersão da argila, foram pesadas $20 \mathrm{~g}$ de solo e adicionados $80 \mathrm{~mL}$ de água, assumindo uma densidade real do solo de 2,65 $\mathrm{g} \mathrm{mL} \mathrm{mL}^{-1}$, gerando assim um volume total de $87,54 \mathrm{~mL}$.

Para a determinação da curva de dispersão de argila, foram aplicados 9 níveis de energia: 0, 100, 200, $300,400,500,600,700$ e $800 \mathrm{~J} \mathrm{~mL}^{-1}$. Para o ponto 100 , foi calculado o tempo de sonicação com base no volume de $87,55 \mathrm{~mL}$, e a potência $93,77 \mathrm{~J} \mathrm{~s}^{-1}$, previamente determinados. Os mesmos cálculos foram realizados para cada um dos níveis de energia, determinando a energia e tempo de sonicação (Tabela 1).

Tabela 1. Tempo necessário para a dispersão de amostra em função da energia requerida.

\begin{tabular}{cccccccccc}
\hline $\mathbf{E}$ & $\mathbf{m L}$ & $\mathbf{J}$ & $\mathbf{J ~ s}^{-1}$ & $\mathbf{s}$ & $\mathbf{m i n}$ & $\mathbf{m i n}$ & $\mathbf{m i n}$ & $\mathbf{s e g}$ & $\mathbf{T}$ \\
\hline 0 & 0 & 0 & 0 & 0 & 0 & 0 & 0 & 0 & 0 \\
100 & 87,55 & 8755 & 93,77 & 93,36 & 1,56 & 1 & 0,56 & 33,60 & 1,34 \\
200 & 87,55 & 17510 & 93,77 & 186,73 & 3,11 & 3 & 0,11 & 6,60 & 3,70 \\
300 & 87,55 & 26265 & 93,77 & 280,10 & 4,67 & 4 & 0,67 & 40,20 & 4,40 \\
400 & 87,55 & 35020 & 93,77 & 373,47 & 6,22 & 6 & 0,22 & 13,20 & 6,13 \\
500 & 87,55 & 43775 & 93,77 & 466,83 & 7,78 & 7 & 0,78 & 46,80 & 7,47 \\
600 & 87,55 & 52530 & 93,77 & 560,20 & 9,34 & 9 & 0,34 & 20,40 & 9,20 \\
700 & 87,55 & 61285 & 93,77 & 653,57 & 10,9 & 12 & 0,47 & 28,20 & 12,28 \\
800 & 87,55 & 70040 & 93,77 & 746,93 & 12,4 & 12 & 0,47 & 28,20 & 12,28 \\
\hline
\end{tabular}

E - energia; $\mathrm{mL}$ - mililitros; J - energia em Joule; s - segundos; min- minutos; T - tempo.

Depois da sonicação, o material de cada nível de energia foi colocado numa proveta de $1000 \mathrm{~mL}$ até atingir o volume máximo. Foi agitado por $1 \mathrm{~min}$ e deixado em repouso por 2 horas para medir a densidade (pelo método de Bouyoucos), mensurando primeiro um branco (proveta com $1000 \mathrm{~mL}$ de água) e a amostra correspondente. Com essas leituras, foi determinado o teor de argila e cada nível de energia (Tabela 2), usando a seguinte equação:

(Teor de argila - Leitura do branco) x $5=$ g de argila 
REVISTA SCIENTIA AGRARIA

Versão On-line ISSN 1983-2443

Versão Impressa ISSN 1519-1125

SA vol. $18 \mathrm{n}^{\circ} .2$ Curitiba Abr/Jun. 2017 p. $22-29$

Tabela 2. Teor de argila

\begin{tabular}{cccc}
\hline Energia $\left(\mathbf{J ~ m L}^{-1}\right)$ & Teor argila (\%) & Branco & Massa argila $(\mathbf{g})$ \\
\hline 0 & 0 & 0 & 0 \\
100 & 0,50 & $-0,50$ & 5,00 \\
200 & 4,50 & 0,50 & 20,00 \\
300 & 5,50 & $-0,50$ & 30,00 \\
400 & 8,00 & 0,00 & 40,00 \\
500 & 10,50 & $-0,50$ & 55,00 \\
600 & 10,50 & $-0,50$ & 55,00 \\
700 & 10,00 & $-1,00$ & 55,00 \\
800 & 10,00 & $-1,00$ & 55,00 \\
\hline
\end{tabular}

\section{Energia, potência e tempo de sonicação}

Com os teores de argila obtidos e os níveis de energia aplicados, foi gerada uma curva de dispersão no programa SIGMAPLOT 12.0 (Figura 3-A).

A curva exponencial tendendo ao máximo não se ajustou aos pontos plotados, então foi realizado um ajuste linear dos primeiros 4 pontos obtendo uma primeira equação parcial (Figura 3-B).

Os últimos 4 pontos também foram ajustados linearmente para obtenção da segunda equação parcial
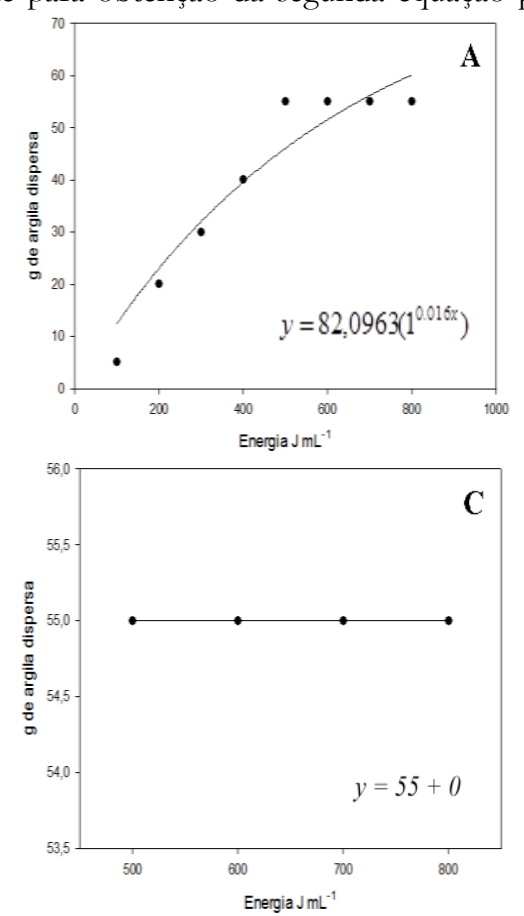

(Figura 3-C). As duas equações parciais foram igualadas para encontrar um ponto de interseção que foi assumido como energia a ser aplicada para a dispersão da amostra do solo do experimento (Figura 3-D).
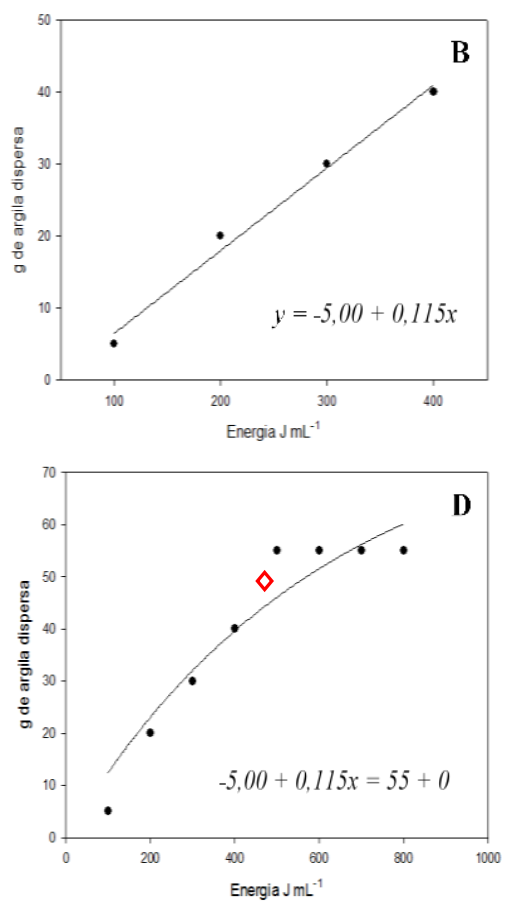

Figura 3. Energia dispersiva para desagregação de microagregados. 3-A. curva exponencial tendendo ao máximo. 3-B. Ajuste linear dos primeiros 4 pontos. 3-C. ajuste linear dos últimos 4 pontos. 3-D. Ponto de interseção de energia a ser aplicada. 


\section{Separação e análise das frações argila e silte}

Após a sonicação, a amostra (por repetição) foi colocada em proveta de $1000 \mathrm{~mL}$, completando um volume até $2 \mathrm{~cm}$ abaixo da parte superior da proveta. A distância percorrida pelas partículas foi de $32,7 \mathrm{~cm}$, e calculada pela lei de Stokes para determinar a velocidade de sedimentação. O tempo assumido de sedimentação segundo a Lei de Stokes foi de 23 horas (82 800 s).

\section{Lei de Stokes}

$v=\frac{G D^{2}(d p-d 1)}{18 n}$

$\mathrm{v}=$ velocidade de sedimentação de partícula

$\mathrm{G}=$ aceleração pela gravidade

$\mathrm{D}=$ diâmetro da partícula

$\mathrm{dp}=$ densidade da partícula

$\mathrm{d} 1$ = densidade da solução (depende da temperatura)

$\mathrm{n}=$ viscosidade da solução

Após as 23 horas, o sobrenadante da amostra foi sifonado e colocado em baldes. Depois da sifonação, foi novamente colocada água na amostra até o mesmo limite ( $2 \mathrm{~cm}$ abaixo da borda superior da proveta) e agitada por um minuto. Este procedimento foi repetido a cada 23 horas por 13 ciclos (13 dias), após dos quais, o sobrenadante ficou transparente.

Para a sedimentação da argila, o sobrenadante total foi floculado com $\mathrm{CaCl}_{2}$, depois, foi colocado em bécker de $1000 \mathrm{~mL}$ para secagem a $50{ }^{\circ} \mathrm{C}$. O mesmo procedimento foi aplicado para o silte.

Após a secagem, as frações foram pesadas para estimar o porcentual de areia, silte e argila para assim determinar a classe textural da amostra. Após esta etapa as amostras foram levadas ao laboratório de Biogeoquímica da DSEA da UFPR para determinação do de COT e NOT, ligados à fração argila e silte pelo método de combustão via seca, por meio do aparelho VARIO EL III - Elementar®.

\section{RESULTADOS E DISCUSSÃO}

As médias das frações areia, silte e argila obtidas de todas as energias de sonicação nas $20 \mathrm{~g}$ de solo analisado, são apresentadas na tabela 3. Os resultados de $\mathrm{C}$ e $\mathrm{N}$ estão apresentados nas figuras 4 e 5 .

Da amostra inicial de $20 \mathrm{~g}$ de solo recuperouse um total de 17,34 g, sendo as frações determinadas com esse valor final, recuperadas.

De acordo com os resultados obtidos no fracionamento físico, o solo analisado pode ser classificado como franco-argiloso, segundo o triângulo de classe textural (SANTOS et al., 2015), característica diretamente relacionada com a posição na paisagem do local, onde foi coletado o solo.

Tabela 3. Frações areia, silte e argila em $20 \mathrm{~g}$ de solo determinadas pelo método de sonicação.

\begin{tabular}{lccc}
\hline Fração & $\begin{array}{c}\text { Média do } \\
\text { peso (g) }\end{array}$ & $\%$ & $\begin{array}{c}\text { Desvio } \\
\text { padrão }\end{array}$ \\
\hline Areia & 4,05 & $23 \%$ & 0,02 \\
Silte & 7,69 & $44 \%$ & 0,10 \\
Argila & 5,60 & $32 \%$ & 0,50 \\
Total recuperado & 17,34 & $100 \%$ & \\
\hline
\end{tabular}

A fração argila apresentou maior capacidade de estocar C e N, seguida das frações silte e areia (Figura 4 e 5). Desta maneira, evidenciou-se que a maior \% de $\mathrm{C}$ está ligada à fração menor que $53 \mu \mathrm{m}$, fração está associada aos minerais, evidenciando a capacidade de interação organo-mineral da fração argila, sendo esta uma das formas de estabilizar e proteger a matéria orgânica no solo.

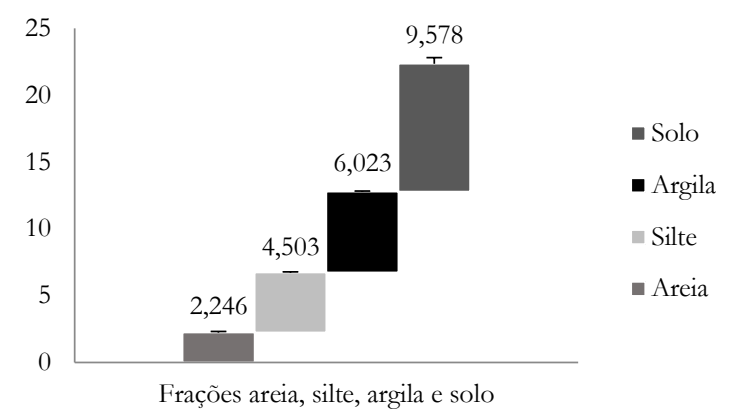

Figura 4. Porcentagem de $\mathrm{C}$ nas diferentes partículas do solo e da amostra inicial.

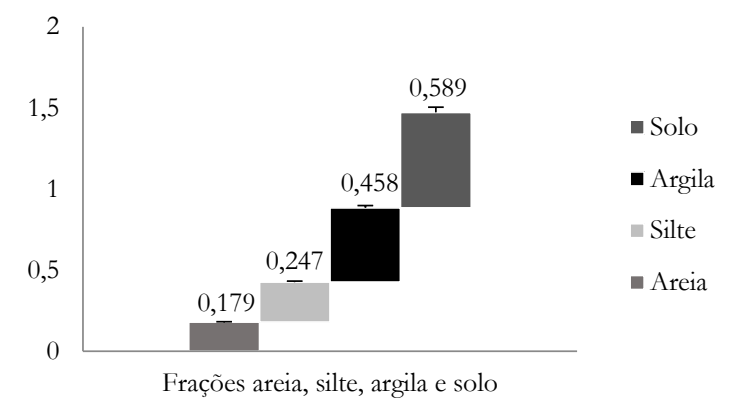

Figura 5. Porcentagem de $\mathbf{N}$ nas diferentes partículas do solo, do solo e da amostra inicial. 
A maior quantidade de $\mathrm{C}$ na fração argila indica que o alto teor de $\mathrm{C}$ deste solo de $90 \mathrm{~g} \mathrm{~kg}^{-1}$ é resultado do alto teor de $\mathrm{C}$ estabilizado nas menores frações de microagregados segundo constatado na SBCS RS/SC (2004). Isso se deve à maior atividade do ânion carboxilato da MOS, coordenando os elementos da estrutura do mineral, através de troca de ligantes (DICK et al., 2009). Já a COP, fração de agregados de 53 - 2000 $\mu \mathrm{m}$, apresentou menores quantidades de $\mathrm{C}$ em relação à COAM (Figura 6). A maior porcentagem de $\mathrm{C}$ associado aos minerais em relação à COP é encontrada tanto para solos de clima temperado (AYOAMA et al., 1999; SLEUTEL et al., 2006; WARING et al, 2014) como para clima tropical (MAFRA, 2013).

A porcentagem total de $\mathrm{C}(9,578 \%)$ e de $\mathrm{N}$ $(0,589 \%)$ no solo sem fracionamento físico foi menor em relação aos fracionados (C- 12,772\% e N - 0,884\%).

Embora a quantidade de $\mathrm{C}$ do COP ter sido menor em relação ao $\mathrm{C}$ da COAM esta fração apresenta relevante importância tanto por servir como uma ponte das frações mais lábeis às mais estabilizadas, como também por apresentar maior sensibilidade indicando quanto o efeito do manejo sobre a área (AYOAMA et al., 1999; MAFRA, 2013).

O papel da COP encontrada neste estudo, embora pouco significativo em relação à COAM, indica que possivelmente esta área vem recebendo contínuo aporte de carbono como resultado do cultivo de plantas C4 (milho) no plano de rotação da área de estudo. A presença de plantas C4 apresentam elevada produção primária líquida (PPL) e tanto os resíduos da parte aérea como das raízes, influenciam na entrada de $\mathrm{C}$ no solo, na forma de biomoléculas de polissacarídeos, que servem como agente cimentante transiente em função de ser um ponto de nucleação tanto para o aumento da atividade microbiana do solo, como para a formação dos microagregados (KUMAR et al., 2013), para posterior estabilização da MOS em um "turnover" de maior duração para a formação de novos microagregados por agentes persistentes (SIX et al., 2004). As contínuas adições de $\mathrm{MO}$, aliadas ao efeito de estabilidade dos macroagregados com tamanho $>250 \mathrm{~m} \mu$, e as repetidas aplicações de estrumes como adubo no solo, possivelmente explicam as proporções de COP encontradas neste estudo. Assim, o contínuo aporte de MO proporcionou a estabilização do $\mathrm{C}$ nos microagregados dos altos teores de $\mathrm{C}$ da COAM encontrados neste estudo.

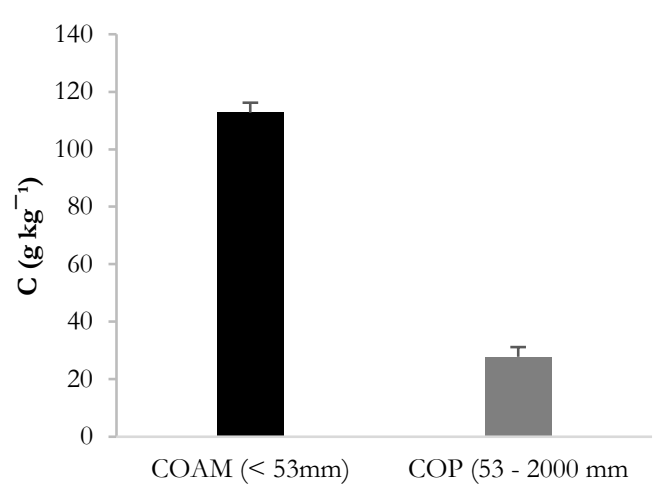

Figura 6. Carbono orgânico particulado (COP) $53-2000$ um e carbono orgânico associado aos minerais (COAM) $<53 \mu \mathrm{m}$.

\section{CONCLUSÃO}

A calibração da sonda pela temperatura da água resultou no aumento da potência com o aumento da temperatura e identificação de amplitude ótima de 50\%;

O maior nível de energia a ser aplicado para esse trabalho foi de $522 \mathrm{~J} \mathrm{~mL}^{-1}$ por 18' e 36" para a amostra de solo com alto teor de MO avaliado;

A maior proporção de $\mathrm{C}$ esteve associada aos minerais e a menor, à fração particulada.

\section{REFERÊNCIAS}

AOYAMA, M; ANGERS, D.A; N'DAYEGAMIYE, A.N. Particulate and mineral - associated organic matter in wáter - stable aggregates as affected by mineral fertilizer and manure applications. Can. J. Soil. Sci. 1999.

BERG, B.; LASKOWSKI, R. Advances in ecological research. Litter decomposition: a guide to carbon and nutrient turnover. Ed. Elserver Academic Press, v.26, p.1-9, 2006.

BURDON, J. Are the traditional concepts of the strutures of humic substances realistic? Soil Science, v.166, n.11, p.752-769, 2001.

DICK, D. P; NOVONTHY, E. H; DIECKOW, J.; BAYER, C. Química da matéria orgânica do solo. In: MELO, V. de F.; ALLEONI, L. R. F. Química $e$ Mineralogia do Solo, 1. ed. Viçosa, MG: SBCS, 2009. v. 1, cap. XI, p.573-610. 
HANK, D. Gênese, interação organo-mineral e estabilidade de agregados de solos desenvolvidos de basalto. Dissertação de mestrado (UFPR), Curitiba- PR, 116 p, 2012.

INDA JUNIOR, A. V.; BAYER, C.; CONCEIÇÃO, P. C.; BOENI, M.; SALTON, J. C.; TONIN, A.T. Variáveis relacionadas à estabilidade de complexos organo-minerais em solos tropicais e subtropicais brasileiros. Ciencia rural, v.37, n.5, p. 1301-1307, 2007.

JAGADAMMA, S.; LAL, R. Distribution of organic carbon in physical fractions of soils as affected by agricultural management. Biol Fertil Soil, n.46, p.543-554, 2010.

KAISER, M.; BERHE, A. A.; SOMMER, M.; KLEBER, M. Application of ultrasound to disperse soil aggregate of high mechanical stability. Journal of Plant Nutrition And Soil Science, n.175, p. 521-526, 2012.

KUMAR, R.; RAWAT, K. S.; SINGH, J.; SINGH, A.; RAI, A. Soil aggregation dynamics and carbon sequestration. Journal of Applied and Natural Science, n.5, p. 250-267, 2013.

LUNARDI, M. H. B. Fracionamento físico da matéria orgânica do solo com diferentes energias de sonicação em três Latossolos Brasileiros sob vegetação nativa. Dissertação de mestrado (UFRGS), Porto Alegre, 2002, 49p.

LUTZOW, M. V.; KOGEL-KNABNER, I.; EKSCHMITT, K.; FLESSA, H.; GUGGENBERGER, G.; MATZNER, E.; MARSCHNER, B. SOM fractionation methods: Relevance to functional pools and to stabilization mechanisms. Soil Biology and Biochemistry, n.39, p. 2183-2207, 2007.

MAFRA, M. S. H. Estoque e fracionamento físico do carbono orgânico em Latossolo sob plantio direto com aplicação de dejeto suíno. Tese de doutorado, Lages- SC, 2013, 88p.

MUSCOLO, A.; SIDARI, M.; NARDI, S.; Humic substance: Relationship between structure and activity. Deeper information suggests univocal findings. Journal of Geochemical Exploration, n.129, p. 57-63, 2013.

PICCOLO, A. The supramolecular structure of humic substances. Soil Science, v.166, n.1, p. 810-832, 2001.
RAINE, S. R; SO, B. An energy based parameter for the assessment of aggregate bond energy. Journal of Soil Science, v. 44, n. 2, p. 249-259, 1993.

SÁ, M. A. C.; LIMA, J. M.; MELLO, C. R. Nível de energia ultra-sônica para o estudo de estabilidade de agregados de um Latossolo sob diferentes usos. Pesquisa Agropecuaria Brasileira., v. 37, n. 11, p. 1649-1655, 2002.

SANTOS, R. D.; SANTOS, H. G; KER, J. C.; ANJOS, L. H. C.; SHIMIZU, S. H. Manual de descrição e coleta de solo no campo. SBCS, Viçosa - MG, 2015, 101p.

SBCS (RS/SC). COMISSÃO DE QUÍMICA E FERTILIDADE DO SOLO. Manual de adubação e calagem para os Estados do Rio Grande do Sul e Santa Catarina. Porto Alegre, 2004.

SCHMIDT, M. W. I.; TORN, M. S.; ABIVEN, S.; DITTMAT, T.; GUGGENBERGER, G.; JANSSENS, I. A.; KLEBER, M.; KOGEL-KNABNER, I.; LEHMANN, J.; MANNING, D. A. C.; NANNIPIERI, P.; RASSE, D. P.; WEINER, S.; TRUMBORE, S. E. Persistence of soil organic matter as an ecosystem property. Nature, v. 478, n.10, p. 49-56, 2011.

SIX, J.; BOSSUYT, H.; DEGRYZE, S.; DENEF, K. A history of research on the link between (micro) aggregates, soil biota and soil organic matter dynamics. Soil \& Tillage Research, n.79, p.7-31, 2004.

SLEUTEL, S. NEVE, S. de; NEMETH, T. TOTH, T; HOFMAN, G. Effect of manure and fertilizer application on the distribution of organic carbono in different soil fractions in long - term field experiments. Europ. J. A, n.25, p. 280-288, 2006.

WARING, B. G.; WEINTRAUB, S. R.; SINSABAUGH, R. L. Ecoenzymatic stoichiometry of microbial nutrient acquisition in tropical soils. Biogeochemistry, DOI: DOI 10.1007/s10533-013-9849-x 